\title{
Successful use of last-option infrapopliteal rotational atherectomy despite microembolisation
}

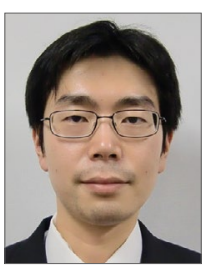

Takahito Doi ${ }^{1}$, MD; Osami Kawarada ${ }^{1,2 *}, \mathrm{MD}, \mathrm{PhD}$; Taka-aki Matsuyama ${ }^{3}, \mathrm{MD}, \mathrm{PhD}$; Shinobu Ayabe ${ }^{4}, \mathrm{MD}$; Hatsue Ishibashi-Ueda ${ }^{3}, \mathrm{MD}, \mathrm{PhD}$; Teruo Noguchi¹, MD, PhD; Hisao Ogawa ${ }^{1}, \mathrm{MD}, \mathrm{PhD}$; Satoshi Yasuda ${ }^{1}, \mathrm{MD}, \mathrm{PhD}$

1. Department of Cardiovascular Medicine, National Cerebral and Cardiovascular Center, Osaka, Japan; 2. Department of Cardiovascular Medicine, Ikuwakai Memorial Hospital, Osaka, Japan; 3. Department of Pathology, National Cerebral and Cardiovascular Center, Osaka, Japan; 4. Department of Plastic Surgery, Yao Tokushukai General Hospital, Osaka, Japan

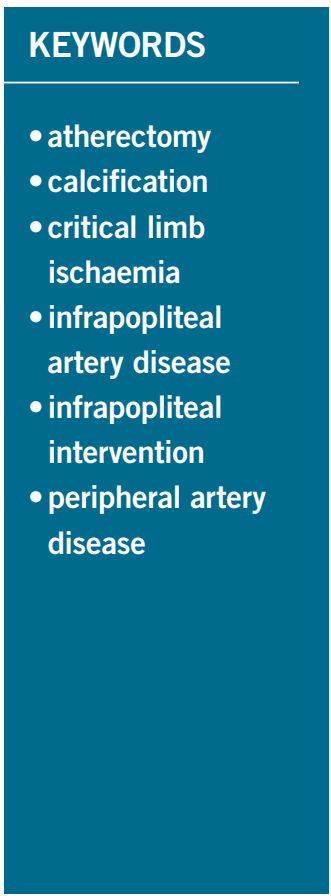

\section{Abstract}

Calcified lesions pose a technical challenge even in contemporary endovascular intervention. A 71-year-old man who had been receiving haemodialysis required infrapopliteal revascularisation for the treatment of ischaemic infectious gangrene of the right toes. Baseline angiography suggested that the multiple stenotic lesions in the anterior tibial artery were amenable to endovascular therapy for the purpose of establishing one straight-line flow to the foot. However, even a $1.25 \times 15 \mathrm{~mm}$ semi-compliant balloon catheter failed to cross and dilate the focal lesion because of the underlying severe calcification in the mid segment of the anterior tibial artery. We adjunctively used high-speed rotational atherectomy with the ROTABLATOR device ( $1.5 \mathrm{~mm}$ burr) to ablate the focal calcified lesion while paying attention to minimise the ablation length and the ablation time. Subsequent balloon angioplasty with a $2.0 \times 40 \mathrm{~mm}$ balloon catheter was successful. The skin perfusion pressure in the right foot increased from 32 to $48 \mathrm{mmHg}$, suggesting a high probability of wound healing. Pathological examination of the right toe amputated on schedule found nonclinically relevant microembolisation involving a couple of cholesterol crystals $(20-30 \mu \mathrm{m})$ located in the arterioles and capillaries of the necrotic tissue. As an adjunctive device, the ROTABLATOR could provide a last resort for limb salvage, albeit that microembolisation can occur.

*Corresponding author: Department of Cardiovascular Medicine, Ikuwakai Memorial Hospital, 3-20-29, Tatsumi-kita, Ikuno-ku, Osaka, 544-0004, Japan.E-mail: osamikawarada@gmail.com 


\section{Introduction}

Given the growing global burden of peripheral artery disease patients with diabetes mellitus and renal failure, calcified lesions pose a technical challenge, even in contemporary endovascular interventions $^{1}$. In the field of coronary intervention, high-speed rotational atherectomy using the ROTABLATORTM (Boston Scientific, Marlborough, MA, USA) device has been used adjunctively for the treatment of calcified lesions for some considerable time. Now might be the prime time to embark on ROTABLATOR use in peripheral interventions in cases of balloon failure, such as unsuccessful crossing or dilatation of a balloon catheter due to underlying severely calcified lesions.

\section{Methods}

A 71-year-old man who had been receiving haemodialysis for 16 years due to chronic nephritis required infrapopliteal revascularisation for the treatment of ischaemic infectious gangrene of the right toes. Skin perfusion pressure (SPP) in the right foot was $32 \mathrm{mmHg}$, even after stenting of the iliac and femoropopliteal artery and surgical endarterectomy of the common femoral artery, suggesting insufficient improvement of the foot circulation for wound healing. Baseline infrapopliteal angiography revealed infrapopliteal artery disease, including multiple stenotic lesions in the anterior tibial artery as well as the affected plantar artery (Figure 1). Based on

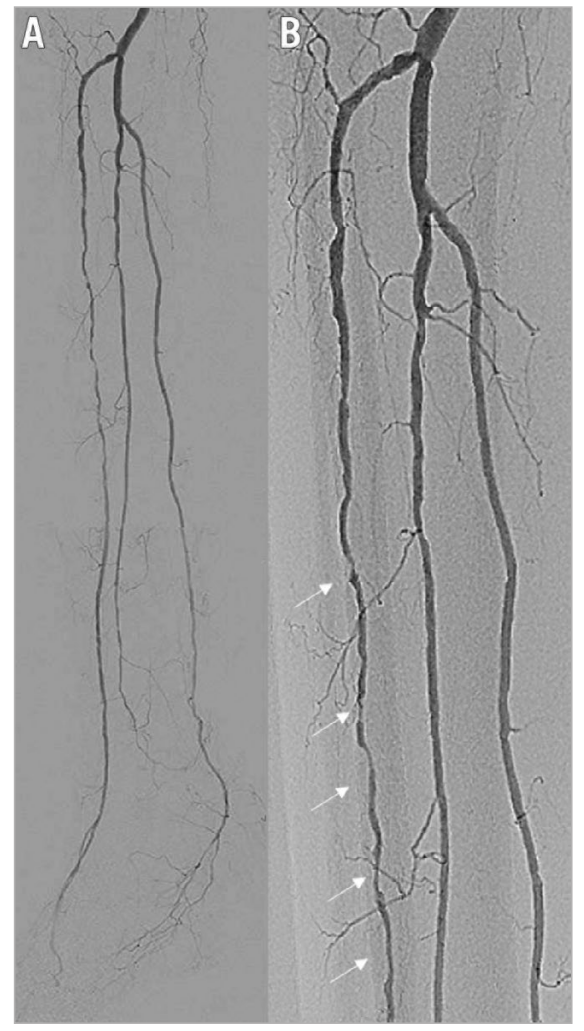

Figure 1. Pre intervention. A) Baseline angiography showing multiple severe stenotic lesions in the anterior tibial artery as well as plantar artery disease. B) Magnified image of multiple severe stenotic lesions in the anterior tibial artery (arrows). our interpretation of the baseline angiogram, stenotic lesions in the anterior tibial artery were amenable to endovascular therapy for the purpose of establishing one straight-line flow to the foot. However, following a successful balloon angioplasty in the proximal segment lesions of the anterior tibial artery, even a $1.25 \times 15 \mathrm{~mm}$ semi-compliant balloon catheter supported by a 4 Fr-long sheath inserted via the ipsilateral common femoral artery failed to cross and dilate the focal stenotic lesion in the mid segment of the anterior tibial artery because of the underlying severe calcification (Figure 2).

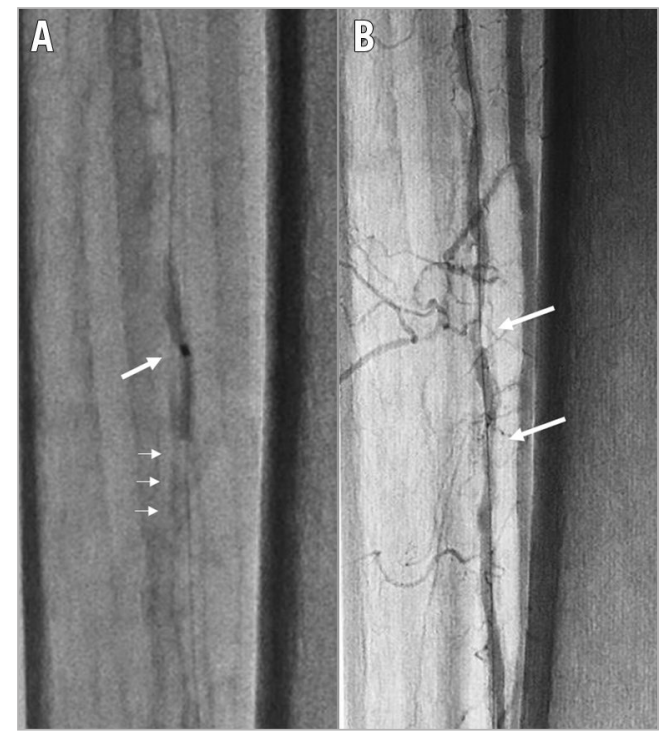

Figure 2. Balloon failure due to underlying calcification in the mid segment of the anterior tibial artery. A) Even a $1.25 \times 15 \mathrm{~mm}$ semi-compliant balloon was stuck just before the calcified lesion (small arrows), and the balloon waist remained due to calcification (large arrow). B) Angiography showing the remaining severe stenosis in the mid segment of the anterior tibial artery (arrows).

We decided to use the ROTABLATOR adjunctively, to facilitate balloon angioplasty. After exchanging the 0.014-inch guidewire for a ROTAWire ${ }^{\mathrm{TM}}$ Floppy (Boston Scientific) using a microcatheter, we focally ablated the calcified lesion using a $1.5 \mathrm{~mm}$ burr while being careful to minimise the ablation length and the ablation time. Subsequent balloon angioplasty with a $2.0 \times 40 \mathrm{~mm}$ balloon catheter was performed successfully (Figure 3 ).

\section{Results}

Final angiography demonstrated the establishment of one straightline flow to the pedal arch (Figure 4). The SPP of the right foot increased to $48 \mathrm{mmHg}$, suggesting a high likelihood of wound healing ${ }^{1}$. Thus, scheduled minor amputation of the right toes was performed to facilitate wound healing and avoid major amputation. Pathological examination of the amputated right toe found non-clinically relevant microembolisation involving a couple of cholesterol crystals $(20-30 \mu \mathrm{m})$ located in the arterioles and capillaries of the necrotic tissue (Figure 5). 


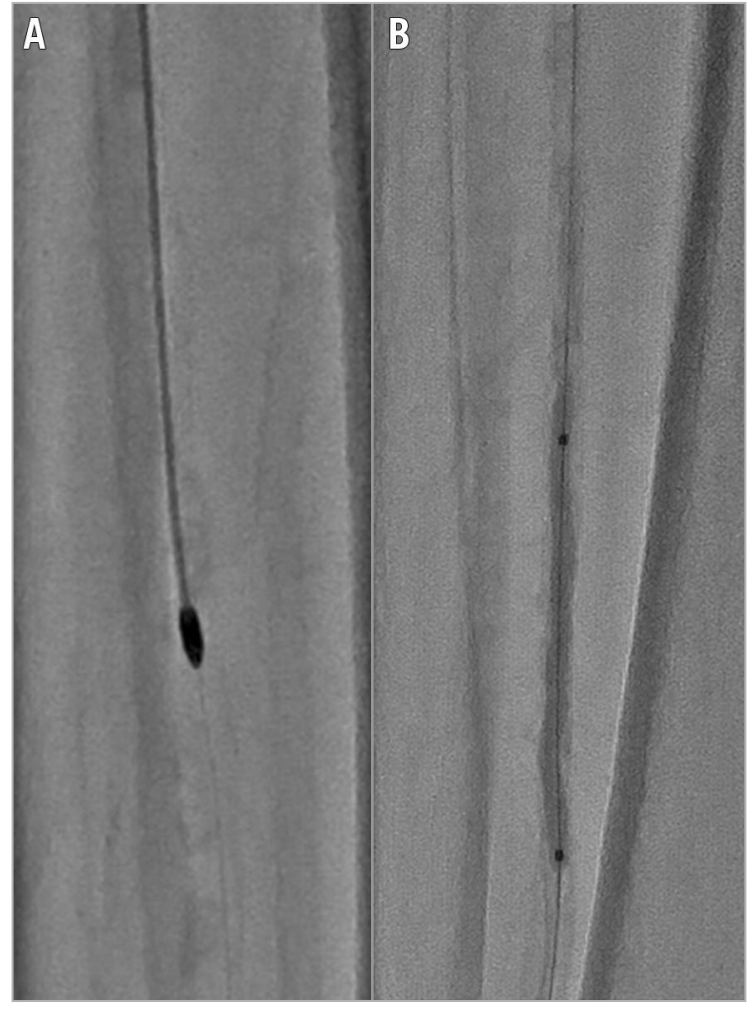

Figure 3. Use of the ROTABLATOR for balloon failure. A) ROTABLATOR with a $1.5 \mathrm{~mm}$ burr was employed to ablate the calcified lesion focally. B) Subsequent balloon angioplasty with a $2.0 \times 40 \mathrm{~mm}$ balloon catheter was successfully performed.

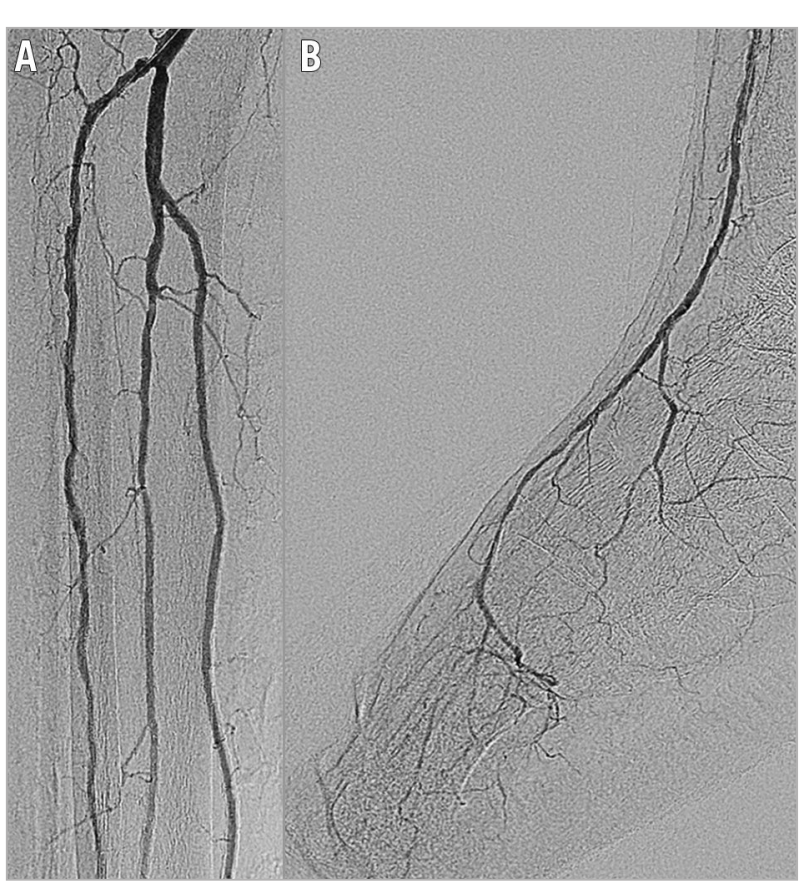

Figure 4. Post intervention. A) Final angiography showing excellent revascularisation. $B$ ) The establishment of one straight-line flow to the pedal arch was achieved. Note the absence of angiographically relevant distal embolisation, even in the below-the-ankle segment.

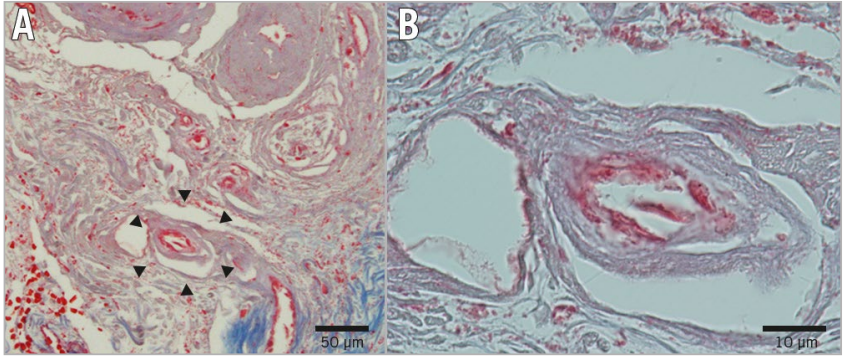

Figure 5. Pathological findings of the amputated right toe.

A) $20 \mu \mathrm{m}$-long cholesterol crystal in the capillaries (arrows).

B) Magnified image of the crystal.

\section{Discussion and limitations}

In the coronary vasculature, the ROTABLATOR has been used as an indispensable adjunctive device to treat severely calcified lesions for over 20 years. However, the role of the ROTABLATOR in the peripheral vasculature has been underappreciated.

According to a multicentre study on peripheral ROTABLATOR use over two decades ago $^{2}$, the angiographic success (residual stenosis $<25 \%$ ) rate of ROTABLATOR use as a standalone procedure in lower limb interventions was approximately $80 \%$ with a variety of complications including haemoglobinuria (13\%), embolisation $(10 \%)$, dissection $(6 \%)$, perforation $(4 \%)$, haematoma $(5 \%)$, infection $(1 \%)$, thrombosis $(11 \%)$, and device-related amputation $(2.5 \%)$. In conjunction with an unacceptably high restenosis rate, enthusiasm for peripheral use of the ROTABLATOR waned. However, recently, there has been increasing interest in using the ROTABLATOR adjunctively in order to facilitate subsequent balloon angioplasty in cases where balloon catheters cannot cross or dilate below-the-knee lesions, because no device can take its place. In a study by Dormal et $\mathrm{al}^{3}$, the ROTABLATOR was used in approximately $10 \%$ (18/183) of procedures, mainly for the treatment of calcified infrapopliteal artery disease that was not suitable for balloon angioplasty alone. The technical success rate of ROTABLATOR use was $100 \%$ (61\% of the cases underwent subsequent balloon angioplasty); the only complication was a case of haemoglobinuria. Despite significant comorbidity $(89 \%$ of cases with critical limb ischaemia, $89 \%$ with very poor run-off, $72 \%$ with diabetes, and $22 \%$ of patients on dialysis), the limb salvage rate was $89 \%$ at 13 -month follow-up. Indeed, as shown in our case, the adjunctive use of the ROTABLATOR device could provide an additional endovascular solution for limb salvage in the clinical setting.

According to a previous experimental investigation ${ }^{4}$, pulverised atherosclerotic particles generated by the use of a ROTABLATOR could theoretically pass harmlessly through the distal microcirculation. However, it is widely recognised that no/slow flow can potentially occur following coronary use of the ROTABLATOR. There has even been a report of a fatal adverse event due to embolisation following the use of a ROTABLATOR in coronary intervention, with a post-mortem autopsy revealing many atherosclerotic fragments in the small arteries, arterioles and capillaries in the territory of the treated coronary artery ${ }^{5}$. 
As with coronary interventions, the potential risk of ROTABLATOR-induced distal embolisation might exist in the setting of critically ischaemic limbs with diminished vascular beds. In our case, we used the ROTABLATOR very focally to minimise the ablation length and the ablation time. Even so, although clinical outcomes were satisfactory, pathological examination found microemboli in the amputated toes. Therefore, when using the ROTABLATOR in the clinical setting, there seems to be a trade-off between blood flow restoration following opening the artery and blood flow disturbance due to microembolisation, even in the affected foot. With this in mind, if the ROTABLATOR is used focally, it should be an indispensable tool even when severely calcified infrapopliteal artery disease does not allow advancement or dilatation of a balloon catheter.

\section{Conclusion}

Adjunctive use of the ROTABLATOR in infrapopliteal revascularisation could provide a last resort for limb salvage in case of balloon failure due to the underlying calcified lesions, albeit that procedure-related microembolisation may occur.

\section{Impact on daily practice}

The ROTABLATOR can be considered as an adjunctive option in cases of balloon failure for the treatment of infrapopliteal artery disease with severely calcified lesions, though the potential of procedure-related microembolisation should always be kept in mind.

\section{Conflict of interest statement}

O. Kawarada reports receiving honoraria for lectures and advisory board fees from Boston Scientific Corporation, and honoraria for lectures and research grants from Terumo. The other authors have no conflicts of interest to declare.

\section{References}

1. Kawarada O, Zen K, Hozawa K, Ayabe S, Huang HL, Choi D, Kim SH, Kim J, Kato T, Tsubakimoto Y, Nakama T, Ichihashi S, Fujimura N, Higashimori A, Fujihara M, Sato T, Yan BP, Pang SY, Wongwanit C, Leong YP, Chua B, George RK, Yokoi Y, Motomura H, Obara H. Contemporary critical limb ischemia: Asian multidisciplinary consensus statement on the collaboration between endovascular therapy and wound care. Cardiovasc Interv Ther. 2018;33:297-312.

2. [No authors listed]. Peripheral atherectomy with the rotablator: a multicenter report. The Collaborative Rotablator Atherectomy Group (CRAG). J Vasc Surg. 1994;19:509-15.

3. Dormal PA, Afrapoli AH, Devaux P. Rotablator: a forgotten tool in limb ischemia? Acta Chir Belg. 2005;105:231-4.

4. Hansen DD, Auth DC, Vracko R, Ritchie JL. Rotational atherectomy in atherosclerotic rabbit iliac arteries. Am Heart J. 1988;115:160-5.

5. Duong van Huyen JP, Fornes P, Iliou MC, Pagny JY, Guermonprez JL, Bruneval P. Fatal coronary embolization following high-speed rotational atherectomy. Histopathology. 1996;29: 73-6. 